TRANSACTIONS OF THE

AMERICAN MATHEMATICAL SOCIETY

Volume 356, Number 8, Pages 3325-3347

S 0002-9947(03)03390-7

Article electronically published on December 15, 2003

\title{
ON THE DIVERGENCE OF THE ROGERS-RAMANUJAN CONTINUED FRACTION ON THE UNIT CIRCLE
}

\author{
DOUGLAS BOWMAN AND JAMES MC LAUGHLIN
}

\begin{abstract}
This paper studies ordinary and general convergence of the RogersRamanujan continued fraction.

Let the continued fraction expansion of any irrational number $t \in(0,1)$ be denoted by $\left[0, e_{1}(t), e_{2}(t), \cdots\right]$ and let the $i$-th convergent of this continued fraction expansion be denoted by $c_{i}(t) / d_{i}(t)$. Let

$$
S=\left\{t \in(0,1): e_{i+1}(t) \geq \phi^{d_{i}(t)} \text { infinitely often }\right\},
$$

where $\phi=(\sqrt{5}+1) / 2$. Let $Y_{S}=\{\exp (2 \pi i t): t \in S\}$. It is shown that if $y \in Y_{S}$, then the Rogers-Ramanujan continued fraction $R(y)$ diverges at $y$. $S$ is an uncountable set of measure zero. It is also shown that there is an uncountable set of points $G \subset Y_{S}$ such that if $y \in G$, then $R(y)$ does not converge generally.

It is further shown that $R(y)$ does not converge generally for $|y|>1$. However we show that $R(y)$ does converge generally if $y$ is a primitive $5 m$-th root of unity, for some $m \in \mathbb{N}$. Combining this result with a theorem of I. Schur then gives that the continued fraction converges generally at all roots of unity.
\end{abstract}

\section{INTRODUCTION}

An infinite continued fraction is an expression of the form

$$
b_{0}+\frac{a_{1}}{b_{1}+\frac{a_{2}}{b_{2}+\frac{a_{3}}{b_{3}+\ldots}} .}
$$

The $a_{i}$ 's and $b_{i}$ 's can be real or complex numbers or functions of one or more variables. To save space, a continued fraction is often written as

$$
b_{0}+\frac{a_{1}}{b_{1}}+\frac{a_{2}}{b_{2}}+\frac{a_{3}}{b_{3}}+\cdots
$$

or as $b_{0}+K_{n=1}^{\infty} a_{n} / b_{n}$. Let $f_{n}$ denote the $n$-th approximant of this continued fraction, namely,

$$
f_{n}=b_{0}+\frac{a_{1}}{b_{1}}+\frac{a_{2}}{b_{2}}+\frac{a_{3}}{b_{3}}+\cdots+\frac{a_{n}}{b_{n}} .
$$

Received by the editors January 17, 2003 and, in revised form, April 15, 2003.

2000 Mathematics Subject Classification. Primary 11A55; Secondary 40A15.

Key words and phrases. Continued fractions, Rogers-Ramanujan.

The second author's research supported in part by a Trjitzinsky Fellowship. 
If the sequence $\left\{f_{n}\right\}_{n=1}^{\infty}$ has a limit, then the continued fraction is said to converge. Otherwise, it is said to diverge.

We also consider $f_{n}$ as a rational function in the variables $a_{1}, \ldots, a_{n}, b_{0}, \ldots, b_{n}$. Let $P_{n}$ denote the numerator and $Q_{n}$ denote the denominator (so that $P_{n}$ is the $n$-th numerator convergent of the continued fraction $b_{0}+K_{n=1}^{\infty} a_{n} / b_{n}$ and $Q_{n}$ is its $n$-th denominator convergent). It is well known (see, for example, $[8$, p.9) that the $P_{n}$ 's and $Q_{n}$ 's satisfy the following recurrence relations:

$$
\begin{gathered}
P_{n}=b_{n} P_{n-1}+a_{n} P_{n-2}, \\
Q_{n}=b_{n} Q_{n-1}+a_{n} Q_{n-2} .
\end{gathered}
$$

It is also well known (see also [8], p.9) that, for $n \geq 1$,

$$
P_{n} Q_{n-1}-P_{n-1} Q_{n}=(-1)^{n-1} \prod_{i=1}^{n} a_{i} .
$$

This paper makes a detailed study of the convergence behaviour of the celebrated Rogers-Ramanujan continued fraction $R(x)$, which is defined for $|x|<1$ by

$$
R(x):=\frac{x^{1 / 5}}{1}+\frac{x}{1}+\frac{x^{2}}{1}+\frac{x^{3}}{1}+\cdots .
$$

Put $K(x)=x^{1 / 5} / R(x)$. It is irrelevant to our work which fifth root is chosen. We are concerned only with questions of convergence, and each approximant to $R(x)$ can be written as $x^{1 / 5} \times r(x)$, where $r(x)$ is some rational function of $x$.

1.1. History. This continued fraction is probably best known through its connection with the famous Rogers-Ramanujan identities:

$$
\begin{aligned}
& \sum_{n=0}^{\infty} \frac{q^{n^{2}}}{(q ; q)_{n}}=\prod_{j=0}^{\infty} \frac{1}{\left(1-q^{5 j+1}\right)\left(1-q^{5 j+4}\right)}, \\
& \sum_{n=0}^{\infty} \frac{q^{n^{2}+n}}{(q ; q)_{n}}=\prod_{j=0}^{\infty} \frac{1}{\left(1-q^{5 j+2}\right)\left(1-q^{5 j+3}\right)},
\end{aligned}
$$

where

$$
(a ; q)_{0}:=1, \quad(a ; q)_{n}=\prod_{j=0}^{n-1}\left(1-a q^{j}\right),|q|<1 .
$$

The connection of these identities with the Rogers-Ramanujan continued fraction is that

$$
K(q)=\frac{\sum_{n=0}^{\infty} \frac{q^{n^{2}}}{(q ; q)_{n}}}{\sum_{n=0}^{\infty} \frac{q^{n^{2}+n}}{(q ; q)_{n}}}, \quad|q|<1 .
$$

The Rogers-Ramanujan identities have a curious history ([5], p. 28). They were first proved by L.J. Rogers in 1894 ([14]) in a paper that was completely ignored. They were rediscovered (without proof) by Ramanujan sometime before 1913. In 1917, Ramanujan rediscovered Roger's paper while browsing a journal. Also in 1917, these identities were rediscovered and proved independently by Issai Schur ([15]). There are now many different proofs. 
Some of the theorems and results in Ramanujan's historic letter to the English mathematician G.H. Hardy in 1913 ([11], p. xxviii) concerned this continued fraction. One of the results from this letter is the following:

$$
R\left(e^{-2 \pi}\right)=\sqrt{\frac{5+\sqrt{5}}{2}}-\frac{\sqrt{5}+1}{2} .
$$

One area of research has been to try to evaluate $R(q)$ for various $q$ inside the unit circle, and indeed many explicit evaluations of $R\left(e^{-\pi \sqrt{n}}\right)$ and $R\left(-e^{-\pi \sqrt{n}}\right)$ have been given for $n \in \mathbb{Q}^{+}$, some of which were asserted by Ramanujan without proof (see, for example, 2], 3], [12] and [16]).

In the same letter, Ramanujan states that if $f:=R(q)$ and $\phi=R\left(q^{5}\right)$, then

$$
f^{5}=\phi \frac{1-2 \phi+4 \phi^{2}-3 \phi^{3}+\phi^{4}}{1+3 \phi+4 \phi^{2}+2 \phi^{3}+\phi^{4}} .
$$

Similar modular equations between $R(q)$ and $R\left(q^{n}\right)$ exist for all $n \in \mathbb{N}$, but not many have been stated explicitly. (Jinhee Yi recently found and proved one in [16], for $n=7$ )

The easiest results on convergence are obtained via the important theorem of Worpitzky (see [8], pp. 35-36), which gives that $R(x)$ converges to a value in $\hat{\mathbb{C}}$ for any $x$ inside the unit circle.

Theorem 1 (Worpitzky). Let the continued fraction $K_{n=1}^{\infty} a_{n} / 1$ be such that $\left|a_{n}\right| \leq$ $1 / 4$ for $n \geq 1$. Then $K_{n=1}^{\infty} a_{n} / 1$ converges. All approximants of the continued fraction lie in the disc $|w|<1 / 2$, and the value of the continued fraction is in the disk $|w| \leq 1 / 2$.

Suppose $|q|>1$. For $n \geq 1$, define

$$
K_{n}(q):=1+\frac{q}{1}+\frac{q^{2}}{1}+\frac{q^{3}}{1}+\cdots+\frac{q^{n}}{1} .
$$

Then

$$
\begin{gathered}
\lim _{j \rightarrow \infty} K_{2 j+1}(q)=\frac{1}{K(-1 / q)}, \\
\lim _{j \rightarrow \infty} K_{2 j}(q)=\frac{K\left(1 / q^{4}\right)}{q} .
\end{gathered}
$$

This was stated by Ramanujan without proof, and was proved by Andrews, Berndt, Jacobsen and Lamphere in 1992 ([1]).

This leaves the question of convergence on the unit circle. Schur showed in 15 that if $x$ is a primitive $m$-th root of unity, where $m \equiv 0(\bmod 5)$, then $K(x)$ diverges, and if $x$ is a primitive $m$-th root of unity, $m \neq \equiv(\bmod 5)$, then $K(x)$ converges and

$$
K(x)=\lambda x^{(1-\lambda \sigma m) / 5} K(\lambda),
$$

where $\lambda=\left(\frac{m}{5}\right)$ (the Legendre symbol) and $\sigma$ is the least positive residue of $m(\bmod 5)$. Note that $(1-\lambda \sigma m) / 5$ is an integer for each $m \neq \equiv 0(\bmod 5)$, and that $K(1)=\phi=(\sqrt{5}+1) / 2$ and $K(-1)=1 / \phi$. It follows that $R(x)$ takes only ten possible values at roots of unity. For later use we define these ten values, $\left\{R_{j}\right\}_{j=1}^{10}$, 
by

$$
R_{j}= \begin{cases}-\phi \exp (2 \pi i j / 5), & 1 \leq j \leq 5 \\ \frac{\exp (2 \pi i j / 5)}{\phi}, & 6 \leq j \leq 10\end{cases}
$$

Remark. Schur's result was essentially proved by Ramanujan, probably earlier than Schur (see [10, p.383). However, he made a calculational error (see [6], p.56).

Summary. The question of convergence for the Rogers-Ramanujan continued fraction has been settled inside the unit circle, outside the unit circle and at roots of unity on the unit circle.

Question. Does the Rogers-Ramanujan continued fraction converge or diverge at a point on the unit circle which is not a root of unity? This has been open since Schur's 1917 paper.

One difficulty. By Schur's result, the unit circle contains a dense set at which $K(q)$ converges and another dense set at which $K(q)$ diverges. Most convergence/divergence criteria depend either on certain inequalities involving the absolute value of the partial quotients or on the partial quotients lying in certain open sets. Obviously neither type of criterion is applicable here.

1.2. Divergence of the Rogers-Ramanujan continued fraction on the unit circle. Here we prove a theorem which shows the existence of an uncountable set of points on the unit circle at which $R(q)$ diverges. We also give explicit examples of such points.

Before stating our theorem, we need to introduce some more notation. Let the regular continued fraction expansion of an irrational $t \in(0,1)$ be denoted $\left[0 ; e_{1}(t), e_{2}(t), e_{3}(t), \ldots\right]$ and let the $i$-th approximant of this continued fraction be denoted $c_{i}(t) / d_{i}(t)$.

We prove the following theorem.

Theorem 2. Let

$$
S=\left\{t \in(0,1): e_{i+1}(t) \geq \phi^{d_{i}(t)} \text { infinitely often }\right\} .
$$

Then $S$ is an uncountable set of measure zero, and if $t \in S$ and $y=\exp (2 \pi i t)$, then $K(y)$ diverges.

An example of a point in $S$ is contained in the following corollary of Theorem 2

Corollary 1. Let $t$ be the number with continued fraction expansion equal to $\left[0, e_{1}, e_{2}, \cdots\right]$, where $e_{i}$ is the integer consisting of a tower of $i$ twos with an $i$ an top. For example,

$$
\begin{aligned}
& t=\left[0,2,2^{2^{2}}, 2^{2^{2^{3}}}, \cdots\right] \\
& =0.484848484848484848484848484848484848484848484848484848484 \\
& \quad 84848484848484848484849277885083112437522992318812011 \cdots . \\
& \text { If } y=\exp (2 \pi i t), \text { then } K(y) \text { diverges. }
\end{aligned}
$$

The key idea here is to construct real numbers $t$ in the interval $(0,1)$, each of which has the property that the sequence of approximants to its continued fraction expansion contains a subsequence of approximants which are "sufficiently close" to 
$t$ in a certain precise sense. (Recall that is possible to construct a real number $t$ for which the $m$-th approximant $c_{m} / d_{m}$ in its continued fraction expansion is as close to $t$ as desired by making the $(m+1)$ st partial quotient $e_{m+1}$ sufficiently large.) If $y:=\exp (2 \pi i t)$ and $x_{m}:=\exp \left(2 \pi i c_{m} / d_{m}\right)$, then $y$ and $x_{m}$ are close enough to keep $Q_{d_{m}-1}(y) Q_{d_{m}-2}(y)$ close to $Q_{d_{m}-1}\left(x_{m}\right) Q_{d_{m}-2}\left(x_{m}\right)$ for the infinite sequence $\left\{d_{m}\right\}_{m=1}^{\infty}$. A result from Schur's paper ([15]) gives that $\left|Q_{d_{m}-1}\left(x_{m}\right) Q_{d_{m}-2}\left(x_{m}\right)\right| \leq$ 4. However, it will be shown that if $K(y)$ converges, then $\lim _{n \rightarrow \infty}\left|Q_{n}(y) Q_{n-1}(y)\right|=$ $\infty$. The fact that $Q_{d_{m}-1}(y) Q_{d_{m}-2}(y)$ stays close to $Q_{d_{m}-1}\left(x_{m}\right) Q_{d_{m}-2}\left(x_{m}\right)$ (which lies in the disc of radius 4 about the origin) for the infinite sequence $\left\{d_{m}\right\}_{m=1}^{\infty}$ means that $\lim _{n \rightarrow \infty}\left|Q_{n}(y) Q_{n-1}(y)\right| \neq \infty$. Thus $K(y)$ does not converge.

1.3. The nature of the non-convergence. An interesting question is what forms can divergence take. In fact there are uncountably many points $y$ on the unit circle such that $R(y)$ has subsequences of approximants tending to all ten of the $R_{j}$ 's defined by (1.5). We prove the following proposition.

Proposition 1. There exists an uncountable set $G^{*}$ of points on the unit circle such that if $y \in G^{*}$ then there exist ten sequences of positive integers, $\left\{n_{i, j}\right\}_{i=1}^{\infty}$, $1 \leq j \leq 10$, such that $\lim _{i \rightarrow \infty} R_{n_{i, j}}(y)=R_{j}$.

Here $\left\{R_{j}\right\}_{j=1}^{10}$ is the set of ten values taken by the Rogers-Ramanujan continued fraction at roots of unity, as defined by (1.5)), and $R_{i}(y)$ is the $i$-th approximant of $R(y)$. This proposition is not strictly necessary for the proof of any of our theorems, but we find the existence of the set $G^{*}$ to be of interest. It is possible to give explicit examples of such points in $G^{*}$. We have the following corollary to Proposition [1.

Corollary 2. Let the sequence of integers $\left\{a_{i}\right\}_{i=1}^{\infty}$ be defined by

$$
\left\{a_{1}, a_{2}, \cdots\right\}=\{0,2, \overline{1,2,1,0,0,1,2,1,0,2,2,4}\},
$$

where the bar indicates that the sequence under it repeats infinitely often. Let $t$ be the number with regular continued fraction expansion given by

$$
t=\left[0, g_{1}+a_{1}, g_{2}+a_{2}, g_{3}+a_{3}, \cdots\right],
$$

where $g_{i}$ is the integer consisting of a tower of $i$ sixteens with an $i$ an top and the $a_{i}$ 's are as above, i.e.,

$$
\begin{aligned}
t= & {\left[0,16,16^{16^{2}}+2,16^{16^{16^{3}}}+1, \cdots\right] } \\
& =0.06249999999999999999999999999999999999999999999999999999999
\end{aligned}
$$

9999999999999999999999999999999999999999999999999999999999999

9999999999999999999999999999999999999999999999999999999999999

9999999999999999999999999999999999999999999999999999999999999

9999999999999999999999999999999999999999999999999999999999999

9999999782707631005156114932594461198007415603592189407975407

$$
1725266194446089419127033011861051603999 \cdots \text {. }
$$

If $y=\exp (2 \pi i t)$, then $R(y)$ has subsequences of approximants tending to all ten values taken by the Rogers-Ramanujan continued fraction at roots of unity. 
1.4. General convergence. In [7], Jacobsen revolutionised the subject of the convergence of continued fractions by introducing the concept of general convergence. General convergence is defined, see [8], as follows.

Let the $n$-th approximant of the continued fraction

$$
M=b_{0}+\frac{a_{1}}{b_{1}+\frac{a_{2}}{b_{2}+\frac{a_{3}}{b_{3}+\ldots}}}
$$

be denoted by $A_{n} / B_{n}$ and let

$$
S_{n}(w)=\frac{A_{n}+w A_{n-1}}{B_{n}+w B_{n-1}} .
$$

Define the chordal metric $d$ on $\hat{\mathbb{C}}$ by

$$
d(w, z)=\frac{|z-w|}{\sqrt{1+|w|^{2}} \sqrt{1+|z|^{2}}}
$$

when $w$ and $z$ are both finite, and

$$
d(w, \infty)=\frac{1}{\sqrt{1+|w|^{2}}}
$$

(The chordal distance $d(w, z)$ between two points $w$ and $z$ in the complex plane is simply half the length of the chord joining their images on the unit sphere centred at the origin, via stereographic projection to the north pole. This metric is used in cases where the point at infinity is not supposed to be singled out as different from other points in the complex plane, as is the case for general convergence.)

Definition. The continued fraction $M$ is said to converge generally to $f \in \hat{\mathbb{C}}$ if there exist sequences $\left\{v_{n}\right\},\left\{w_{n}\right\} \subset \hat{\mathbb{C}}$ such that $\lim \inf d\left(v_{n}, w_{n}\right)>0$ and

$$
\lim _{n \rightarrow \infty} S_{n}\left(v_{n}\right)=\lim _{n \rightarrow \infty} S_{n}\left(w_{n}\right)=f .
$$

Remark. Jacobsen shows in [7] that, if a continued fraction converges in the general sense, then the limit is unique.

The idea of general convergence is of great significance because classical convergence implies general convergence (take $v_{n}=0$ and $w_{n}=\infty$, for all $n$ ), but the converse does not necessarily hold. If a continued fraction converges in the general sense, but not in the classical sense, computing the limit in the general sense is just as easy as computing the limit for continued fractions that converge in the classical sense, i.e., it is computationally effective. Moreover, general convergence is a useful method of accelerating the convergence of continued fractions which converge already in the classical sense. In summary, general convergence is a natural extension of the concept of classical convergence for continued fractions.

In regard to the Rogers-Ramanujan continued fraction, some natural questions are the following:

1) Does $K(q)$ converge generally if $q$ is an $m$-th root of unity and $5 \mid m$ ?

2) Does $K(q)$ converge generally if $|q|>1$ ?

3) Does $K(q)$ converge generally if $|q|=1$ and $q$ is not a root of unity?

We will show that the Rogers-Ramanujan continued fraction converges generally at primitive $5 m$-th roots of unity, for each $m \in \mathbb{N}$. This is in contrast to classical 
convergence, where the Rogers-Ramanujan continued fraction diverges at primitive $5 m$-th roots of unity. We further show that the Rogers-Ramanujan continued fraction does not converge generally outside the unit circle. We also have the following theorem on divergence in the general sense on the unit circle. (For details see Theorem 4 in Section 5 )

Theorem 4. There is an uncountable constructible set $G$ of points on the unit circle such that if $y \in G$, then $K(y)$ does not converge generally.

We also show that when $y$ is the point in Corollary 1 above, the Rogers-Ramanujan continued fraction does not converge in the general sense either.

Corollary 3. Let $y$ be as in Corollary 1 , Then $K(y)$ does not converge generally.

\section{Divergence in the Classical Sense on the unit Circle}

Before proving Theorem 2 we need some preliminary results. For $n \geq 1$, let

$$
K_{n}(x):=1+K_{j=1}^{n} \frac{x^{j}}{1}=\frac{P_{n}(x)}{Q_{n}(x)}
$$

denote the $n$-th approximant of $K(x)$. It follows from (1.2) that if $|x|=1$, then, for $n \geq 1$,

$$
\left|P_{n}(x) Q_{n-1}(x)-Q_{n}(x) P_{n-1}(x)\right|=1 .
$$

Suppose $K(q)$ converges to $L \in \mathbb{C}$ at $q=y$, so that $\lim _{n \rightarrow \infty} P_{n}(y) / Q_{n}(y)=L$. Then

$$
\left|\frac{P_{n}(y)}{Q_{n}(y)}-\frac{P_{n-1}(y)}{Q_{n-1}(y)}\right| \leq\left|\frac{P_{n}(y)}{Q_{n}(y)}-L\right|+\left|\frac{P_{n-1}(y)}{Q_{n-1}(y)}-L\right| .
$$

It follows that

$$
0=\lim _{n \rightarrow \infty}\left|\frac{P_{n}(y)}{Q_{n}(y)}-\frac{P_{n-1}(y)}{Q_{n-1}(y)}\right|=\lim _{n \rightarrow \infty}\left|\frac{P_{n}(y) Q_{n-1}(y)-P_{n-1}(y) Q_{n}(y)}{Q_{n}(y) Q_{n-1}(y)}\right| .
$$

Thus by (2.1) and (2.2) it follows that if $K(y)$ converges, then

$$
\lim _{n \rightarrow \infty} Q_{n}(y) Q_{n-1}(y)=\infty .
$$

We will exhibit an uncountable set of points on the unit circle for which this fails to happen.

It follows from the recurrence relations at (1.1) that

$$
Q_{n+1}(x)=Q_{n}(x)+x^{n+1} Q_{n-1}(x) .
$$

It follows easily from (2.3), the triangle inequality and a simple induction that, for $n \geq 2$,

$$
\left|Q_{n}(x)\right| \leq F_{n+1},
$$

where $\left\{F_{i}\right\}_{i=1}^{\infty}$ denotes the Fibonacci sequence defined by $F_{1}=F_{2}=1$ and $F_{i+1}=$ $F_{i}+F_{i-1}$. For the following lemma, we recall that the regular continued fraction expansion of an irrational number $t \in(0,1)$ is denoted $\left[0 ; e_{1}(t), e_{2}(t), e_{3}(t), \ldots\right]$ and that the $i$-th approximant of this continued fraction is denoted $c_{i}(t) / d_{i}(t)$. 
Lemma 1. With the notation of Theorem 圆 for $t \in S$, we have

$$
\left|t-\frac{c_{i}(t)}{d_{i}(t)}\right|<\frac{1}{d_{i}(t)^{2} \phi^{d_{i}(t)}}
$$

for infinitely many $i$.

Proof. Let $i$ be one of the infinitely many integers for which $e_{i+1}(t) \geq \phi^{d_{i}(t)}$, and let $t_{i+1}=\left[e_{i+1}(t), e_{i+2}(t), \ldots\right]$ denote the $i$-th tail of the continued fraction expansion for $t$. Then

$$
\begin{aligned}
\left|t-\frac{c_{i}(t)}{d_{i}(t)}\right| & =\left|\frac{t_{i+1} c_{i}(t)+c_{i-1}(t)}{t_{i+1} d_{i}(t)+d_{i-1}(t)}-\frac{c_{i}(t)}{d_{i}(t)}\right| \\
& =\frac{1}{d_{i}(t)\left(t_{i+1} d_{i}(t)+d_{i-1}(t)\right)} \\
& <\frac{1}{d_{i}(t)\left(e_{i+1}(t) d_{i}(t)+d_{i-1}(t)\right)}<\frac{1}{d_{i}(t)^{2} \phi^{d_{i}(t)}} .
\end{aligned}
$$

Lemma 21 Let $x$ and $y$ be two points on the unit circle. Then, for all integers $n \geq 0$,

$$
\left|Q_{n}(x)-Q_{n}(y)\right| \leq n^{2} \phi^{n}|x-y|
$$

and

$$
\left|P_{n}(x)-P_{n}(y)\right| \leq(n+1)^{2} \phi^{n+1}|x-y| .
$$

Proof. The assertions of the lemma can easily be checked for $n=0,1$.

Let $\beta_{i}=\left|Q_{i}(x)-Q_{i}(y)\right|$ and $\delta_{i}=(i+1) F_{i}|x-y|$. From (2.3) and (2.4) it easily follows that

$$
\beta_{n} \leq \beta_{n-1}+\beta_{n-2}+\delta_{n-1} .
$$

We now claim that, for $r=2, \ldots, n-1$,

$$
\beta_{n} \leq F_{r} \beta_{n-r+1}+F_{r-1} \beta_{n-r}+\sum_{i=1}^{r-1} F_{i} \delta_{n-i} .
$$

The claim is true for $r=2$ by (2.7). Suppose it is true for $r=2, \ldots, s$. Then

$$
\begin{aligned}
\beta_{n} & \leq F_{s} \beta_{n-s+1}+F_{s-1} \beta_{n-s}+\sum_{i=1}^{s-1} F_{i} \delta_{n-i} \\
& \leq F_{s}\left(\beta_{n-s}+\beta_{n-s-1}+\delta_{n-s}\right)+F_{s-1} \beta_{n-s}+\sum_{i=1}^{s-1} F_{i} \delta_{n-i} \\
& =F_{s+1} \beta_{n-s}+F_{s} \beta_{n-s-1}+\sum_{i=1}^{s} F_{i} \delta_{n-i},
\end{aligned}
$$

and (2.8) is true by induction for $2 \leq r \leq n-1$.

\footnotetext{
${ }^{1}$ The authors wish to thank the referee for pointing out that Lemma 2 may also be obtained from Bernstein's inequalities for trignometric polynomials (Theorems 1.2.3, 1.2.4, pp. 531-532 of [9], or in [4]).
} 
Recall that $\beta_{1}=0$ and $\beta_{2}=\left|\left(1+x^{2}\right)-\left(1+y^{2}\right)\right| \leq 2|x-y|$. Now in (2.8) let $r=n-1$. This gives

$$
\begin{aligned}
\beta_{n} & \leq 2 F_{n-1}|x-y|+\sum_{i=1}^{n-2} F_{i} \delta_{n-i}=\sum_{i=1}^{n-1} F_{i}(n-i+1) F_{n-i}|x-y| \\
& \leq \sum_{i=1}^{n-1} \phi^{n}(n-i+1)|x-y| .
\end{aligned}
$$

The last inequality uses the bound $F_{j} \leq \phi^{j}$. This last expression simplifies to give

$$
\beta_{n} \leq \phi^{n}|x-y| \sum_{i=2}^{n} i<n^{2} \phi^{n}|x-y| .
$$

Statement (2.6) follows similarly.

To show our set $S$ has measure zero, we use the following lemma.

Lemma 3 ([13]). Let $f(n)>1$ for $n=1,2, \cdots$, and suppose $\sum_{n=1}^{\infty} 1 / f(n)<\infty$. Then the set $S^{*}=\left\{t \in(0,1): e_{k}(t)>f(k)\right.$ infinitely often $\}$ has measure zero.

We now prove the existence of uncountably many points on the unit circle at which $K(q)$ diverges.

\section{Theorem 2, Let}

$$
S=\left\{t \in(0,1): e_{i+1}(t) \geq \phi^{d_{i}(t)} \text { infinitely often }\right\} .
$$

Then $S$ is an uncountable set of measure zero, and if $t \in S$ and $y=\exp (2 \pi i t)$, then $K(y)$ diverges.

Proof. Let $t \in S$ with approximants $\left\{c_{n} / d_{n}\right\}_{n=0}^{\infty}$. Let $y=\exp (2 \pi i t)$ and let $x_{n}=$ $\exp \left(2 \pi i c_{n} / d_{n}\right)$. Schur showed in [15] that if $x$ is a primitive $m$-th root of unity, then, depending on the congruence class of $m(\bmod 5), Q_{m-1}(x)$ and $Q_{m-2}(x)$ are each either 0,1 , a root of unity or the sum of two roots of unity (see Table 1). It follows that

$$
\max \left\{\left|Q_{d_{n}-1}\left(x_{n}\right)\right|,\left|Q_{d_{n}-2}\left(x_{n}\right)\right|\right\} \leq 2 .
$$

TABLE 1.

\begin{tabular}{|c|c|c|c|c|}
\hline$m$ & $P_{m-2}$ & $P_{m-1}$ & $Q_{m-2}$ & $Q_{m-1}$ \\
\hline $5 \mu$ & 0 & $-x^{2 m / 5}-x^{-2 m / 5}$ & $-x^{2 m / 5}-x^{-2 m / 5}$ & 0 \\
$5 \mu+1$ & $x^{(1-m) / 5}$ & 1 & 0 & $x^{(-1+m) / 5}$ \\
$5 \mu-1$ & $x^{(1+m) / 5}$ & 1 & 0 & $x^{(-1-m) / 5}$ \\
$5 \mu+2$ & $-x^{(1+2 m) / 5}$ & 0 & 1 & $-x^{(-1-2 m) / 5}$ \\
$5 \mu-2$ & $-x^{(1-2 m) / 5}$ & 0 & 1 & $-x^{(-1+2 m) / 5}$ \\
\hline
\end{tabular}


We use, in turn, Lemma 2 the fact that chord length is shorter than arc length, and Lemma 1 to get that, for infinitely many $n$,

$$
\begin{aligned}
\left|Q_{d_{n}-1}\left(x_{n}\right)-Q_{d_{n}-1}(y)\right| & \leq\left(d_{n}-1\right)^{2} \phi^{d_{n}-1}\left|x_{n}-y\right| \\
& <\left(d_{n}-1\right)^{2} \phi^{d_{n}-1} 2 \pi\left|\frac{c_{n}}{d_{n}}-t\right| \\
& <\left(\frac{d_{n}-1}{d_{n}}\right)^{2} \frac{2 \pi}{\phi}<4 .
\end{aligned}
$$

Similarly,

$$
\left|Q_{d_{n}-2}\left(x_{n}\right)-Q_{d_{n}-2}(y)\right|<\left(\frac{d_{n}-2}{d_{n}}\right)^{2} \frac{2 \pi}{\phi^{2}}<4 .
$$

We apply the triangle inequality to (2.11) and (2.12) and use (2.10) to get that $\left|Q_{d_{n}-1}(y)\right|<6$ and $\left|Q_{d_{n}-2}(y)\right|<6$. Finally, we deduce that

$$
\left|Q_{d_{n}-1}(y) Q_{d_{n}-2}(y)\right|<36 \text {. }
$$

Since this holds for infinitely many terms of the sequence $\left\{d_{n}\right\}_{n=0}^{\infty}$, it follows that

$$
\lim _{n \rightarrow \infty} Q_{n}(y) Q_{n-1}(y) \neq \infty,
$$

and thus $K(y)$ does not converge.

We next show that $S$ has measure zero (it is clearly an uncountable set). Let $f(i)=\phi^{F_{i}}, i=1,2, \cdots$. Then it follows that $\sum_{i=1}^{\infty} 1 / f(i)<\infty$. Let

$$
S^{*}=\left\{t \in(0,1): e_{i+1}(t) \geq \phi^{F_{i+1}} \quad \text { infinitely often }\right\},
$$

so by Lemma(3), $S^{*}$ has measure zero.

Recall that $S=\left\{t \in(0,1): e_{i+1}(t) \geq \phi^{d_{i}(t)}\right.$ infinitely often $\}$. We have $S \subset S^{*}$, since $d_{i}(t) \geq F_{i+1}$, and thus $S$, being a subset of a set of measure zero, has measure zero.

For later use, we define $Y_{S}=\{\exp (2 \pi i t): t \in S\}$, where $S$ is the set defined in Theorem 2

Corollary 1, Let $t$ be the number with continued fraction expansion equal to $\left[0, e_{1}, e_{2}, \cdots\right]$, where $e_{i}$ is the integer consisting of a tower of $i$ twos with an $i$ an top. For example,

$$
t=\left[0,2,2^{2^{2}}, 2^{2^{2^{3}}}, \cdots\right] .
$$

If $y=\exp (2 \pi i t)$, then $K(y)$ diverges.

Proof. Denote the $i$-th approximant of the continued fraction expansion of $t$ by $c_{i} / d_{i}$. We will show that, for $i=1,2, \ldots$,

$$
e_{i+1} \geq 2^{d_{i}}>\phi^{d_{i}} \text {. }
$$

Then $K(y)$ diverges by Theorem [2. By the definition of the $e_{i}$ 's, we have that

$$
2^{d_{i}} \leq e_{i+1} \text { if and only if } d_{i} \leq \underbrace{2^{2^{2+1}}}_{i \text { twos }},
$$


where the notation indicates that the last integer consists of a tower of $i$ twos with an $i+1$ on top. It can be easily checked that the second inequality holds for $i=1,2$. Suppose it holds for $i=1,2, \ldots, r-1$. Then

$$
\begin{gathered}
d_{r}=e_{r} d_{r-1}+d_{r-2} \leq \underbrace{2^{2}}_{r \text { twos }} \times \underbrace{2^{r}}_{(r-1) \text { twos }}+\underbrace{2^{2}}_{(r-2) \text { twos }} \\
\leq \underbrace{2^{2^{r}}}_{r \text { twos }} .
\end{gathered}
$$

Thus the first inequality in (2.14) holds for all positive integers $i$, and the result follows.

\section{General convergence at $5 m$ th Roots of unity}

We next consider the question of general convergence for the Rogers-Ramanujan continued fraction when $q$ is a primitive $m$-th root of unity, where $m \equiv 0(\bmod 5)$. As Schur showed in [15], $K(x)$ does not converge in the classical sense in this case. We have the following proposition.

Proposition 2. If $x$ is an $m$-th root of unity, where $m \equiv 0(\bmod 5)$, then $K(x)$ converges generally.

Proposition 2 along with Schur's theorem shows that $K(x)$ converges generally at any root of unity.

Proof of Proposition Q. From [15, for $0 \leq r<m$,

$$
P_{q m+r}=P_{r} P_{m-1}^{q}, \quad Q_{q m+r}=Q_{r} Q_{m-2}^{q} .
$$

Also from [15] (see Table 1),

$$
P_{m-1}=-x^{2 m / 5}-x^{-2 m / 5}, \quad Q_{m-2}=-x^{m / 5}-x^{-m / 5} .
$$

Let $\left\{u_{n}\right\}_{n=1}^{\infty}$ be a sequence in $\hat{\mathbb{C}}$. It is convenient to separate $n \in \mathbb{Z}^{+}$into residue classes modulo $m$. We put $n=q m+r$. From (3.1),

$$
S_{n}\left(u_{n}\right)=\frac{P_{n}+u_{n} P_{n-1}}{Q_{n}+u_{n} Q_{n-1}}= \begin{cases}\left(\frac{P_{m-1}}{Q_{m-2}}\right)^{q} \frac{P_{r}+u_{n} P_{r-1}}{Q_{r}+u_{n} Q_{r-1}}, & 1 \leq r \leq m-1, \\ \left(\frac{P_{m-1}}{Q_{m-2}}\right)^{q} \frac{P_{m-1}+u_{n} P_{m-1}}{Q_{m-2}+u_{n} Q_{m-1}}, & r=m .\end{cases}
$$

Suppose that $x^{m / 5}$ is in the second or third quadrant. Then

$$
\left|P_{m-1}\right|=2 \cos \left(\frac{2 \pi}{5}\right)=\frac{\sqrt{5}-1}{2}<\left|Q_{m-2}\right|=2 \cos \left(\frac{\pi}{5}\right)=\frac{\sqrt{5}+1}{2} .
$$

Hence

$$
\left|\frac{P_{m-1}}{Q_{m-2}}\right|<1
$$


We now construct two sequences $\left\{v_{n}\right\}$ and $\left\{w_{n}\right\}$ which satisfy the conditions for general convergence at $x$. Let

$$
M=\max _{1 \leq r \leq m}\left\{\left|\frac{Q_{r}}{Q_{r-1}}\right|: Q_{r-1} \neq 0\right\} .
$$

Put $v_{n}=M+1$ and $w_{n}=M+2$, for $n=1,2, \ldots$ Hence $\liminf d\left(v_{n}, w_{n}\right)>0$, and by (3.2) and (3.3),

$$
\lim _{n \rightarrow \infty} \frac{P_{n}+v_{n} P_{n-1}}{Q_{n}+v_{n} Q_{n-1}}=\lim _{n \rightarrow \infty} \frac{P_{n}+w_{n} P_{n-1}}{Q_{n}+w_{n} Q_{n-1}}=0 .
$$

Thus $K(x)$ converges generally to 0 in this case.

Next suppose that $x^{m / 5}$ is in the first or fourth quadrant, so that

$$
\left|P_{m-1}\right|=2 \cos \left(\frac{\pi}{5}\right)>\left|Q_{m-2}\right|=2 \cos \left(\frac{2 \pi}{5}\right) .
$$

Then

$$
\left|\frac{P_{m-1}}{Q_{m-2}}\right|>1
$$

In this case let

$$
M=\max _{1 \leq r \leq m}\left\{\left|\frac{P_{r}}{P_{r-1}}\right|: P_{r-1} \neq 0\right\} .
$$

As before, let $v_{n}=M+1$ and $w_{n}=M+2$, for $n=1,2, \ldots$ Hence $\liminf d\left(v_{n}, w_{n}\right)$ $>0$, and by (3.2) and (3.4),

$$
\lim _{n \rightarrow \infty} \frac{P_{n}+v_{n} P_{n-1}}{Q_{n}+v_{n} Q_{n-1}}=\lim _{n \rightarrow \infty} \frac{P_{n}+w_{n} P_{n-1}}{Q_{n}+w_{n} Q_{n-1}}=\infty .
$$

Thus $K(x)$ converges generally to $\infty$ in the second case.

\section{General convergence outside the unit circle}

We next consider the question of general convergence for $K(q)$ outside the unit circle. It was proved in [1] that if $0<|x|<1$, then the odd approximants of $1 / K(1 / x)$ tend to

$$
1-\frac{x}{1}+\frac{x^{2}}{1}-\frac{x^{3}}{1}+\cdots:=F_{1}(x)
$$

while the even approximants tend to

$$
\frac{x}{1}+\frac{x^{4}}{1}+\frac{x^{8}}{1}+\frac{x^{12}}{1}+\cdots:=F_{2}(x) .
$$

By Worpitzky's theorem each continued fraction does converge inside the unit circle to values in $\hat{\mathbb{C}}$. It is not clear from (4.1) and (4.2) that $F_{1}(x) \neq F_{2}(x)$ for all $x$ inside the unit circle. However, it is an easy consequence of the Stern-Stolz Theorem that $K(q)$ diverges in the general sense for $|q|>1$.

Theorem 3 (The Stern-Stolz Theorem [8], p. 94). The continued fraction $b_{0}+$ $K_{n=1}^{\infty}\left(1 / b_{n}\right)$ diverges generally if $\sum\left|b_{n}\right|<\infty$. In fact,

$$
\lim _{n \rightarrow \infty} A_{2 n+p}=P_{p} \neq \infty, \quad \lim _{n \rightarrow \infty} B_{2 n+p}=Q_{p} \neq \infty,
$$

for $p=0,1$, where

$$
P_{1} Q_{0}-P_{0} Q_{1}=1
$$


After an equivalence transformation to put $K(q)$ in the form in the statement of Theorem [3] we get that

$$
K(q)=1+\frac{1}{1 / q}+\frac{1}{1 / q}+\frac{1}{1 / q^{2}}+\frac{1}{1 / q^{2}}+\frac{1}{1 / q^{3}}+\frac{1}{1 / q^{3}}+\cdots
$$

It is clear that if $|q|>1$, then $\sum\left|b_{n}\right|<\infty$, giving that $K(q)$ does not converge in the general sense.

\section{General convergence on the unit circle}

In this section we prove the following theorem.

Theorem 4. Let $t$ be any irrational in $(0,1)$ for which there exist two subsequences of approximants $\left\{c_{f_{n}} / d_{f_{n}}\right\}$ and $\left\{c_{g_{n}} / d_{g_{n}}\right\}$, integers $r, u \in\{0,1,2,3,4\}$, and integers $s, v \in\{1,2,3,4\}$ such that

$$
\begin{aligned}
c_{f_{n}} \equiv r(\bmod 5), & c_{g_{n}} \equiv u(\bmod 5), \\
d_{f_{n}} \equiv s(\bmod 5), & d_{g_{n}} \equiv v(\bmod 5) .
\end{aligned}
$$

and

$$
e_{h_{n}+1}(t)>2 \pi\left(d_{h_{n}}+1\right)^{2} \phi^{d_{h_{n}}^{2}+2 d_{h_{n}}},
$$

for all $n$, where $h_{n}=f_{n}$ or $g_{n}$.

Suppose further that

$$
R(\exp (2 \pi i r / s))=R_{a} \neq R_{b}=R(\exp (2 \pi i u / v)),
$$

for some $a, b \in\{1,2, \cdots, 10\}$. Let $S^{\diamond}$ denote the set of all $t \in(0,1)$ satisfying (5.1), (5.2) and (5.3), and set

$$
G=\left\{\exp (2 \pi i t): t \in S^{\diamond}\right\} .
$$

Then $G$ is an uncountable set such that if $y \in G$, then $K(y)$ does not converge generally.

Before proving Theorem 4, it is necessary to prove some technical lemmas. In what follows, $x$ is a primitive $m$-th root of unity, where $m \neq \equiv(\bmod 5), \bar{\phi}=$ $(-\sqrt{5}+1) / 2, K_{j}=K_{j}(x), P_{j}=P_{j}(x)$ and $Q_{j}=Q_{j}(x)$, for $j=0,1,2, \ldots$ Frequent use will be made of Binet's formula for $F_{k}$, namely,

$$
F_{k}=\frac{\phi^{k}-\bar{\phi}^{k}}{\sqrt{5}}
$$

Note that $\lim _{k \rightarrow \infty} F_{k+1} / F_{k}=\phi$.

We also use the following facts, some of which can be found in [15] and some of which can be deduced from Table 1 (also from [15]). For $n \geq m, q \geq 1$ and $0 \leq r<m$, we have the following equalities:

$$
\begin{gathered}
P_{n}=P_{m-1} P_{n-m}+P_{m-2} Q_{n-m}, \\
Q_{n}=Q_{m-1} P_{n-m}+Q_{m-2} Q_{n-m}, \\
P_{q m+r}=P_{(q-1) m+r}+P_{(q-2) m+r}, \\
Q_{q m+r}=Q_{(q-1) m+r}+Q_{(q-2) m+r} .
\end{gathered}
$$


For $0 \leq r<m$, there exist constants $b_{r}$ and $b_{r}^{\prime}$ such that

$$
\begin{gathered}
Q_{q m+r}=b_{r} \phi^{q}+b_{r}^{\prime} \bar{\phi}^{q}, \\
Q_{2 m-1}=Q_{m-1}, \quad P_{2 m-1}=P_{m-1}+1, \\
P_{2 m-2}=P_{m-2}, \quad Q_{2 m-2}=1+Q_{m-2} .
\end{gathered}
$$

Lemma 4. For $q \geq 2$,

$$
\begin{gathered}
\phi^{q-1} \leq\left|Q_{q m+m-1}\right| \leq \phi^{q}, \\
\text { If } m \equiv 1,-1(\bmod 5), \text { then } \phi^{q-2} \leq\left|Q_{q m+m-2}\right| \leq \phi^{q-1}, \\
\text { If } m \equiv 2,-2(\bmod 5), \text { then } \phi^{q} \leq\left|Q_{q m+m-2}\right| \leq \phi^{q+1}, \\
\frac{1}{\phi^{2}} \leq\left|\frac{Q_{q m+m-1}}{Q_{q m+m-2}}\right| \leq \phi^{2} .
\end{gathered}
$$

Proof. From (5.8) and (5.9) it follows that

$$
Q_{q m+m-1}=\frac{Q_{m-1}}{\sqrt{5}}\left(\phi^{q+1}-\bar{\phi}^{q+1}\right)=\phi^{q+1} \frac{Q_{m-1}}{\sqrt{5}}\left(1-\frac{(-1)^{q+1}}{\phi^{2 q+2}}\right) .
$$

From Table 1, $\left|Q_{m-1}\right|=1$, and since $q \geq 2$, it follows that

$$
\frac{\phi^{q+1}}{\sqrt{5}}\left(1-\frac{1}{\phi^{6}}\right) \leq\left|Q_{q m+m-1}\right| \leq \frac{\phi^{q+1}}{\sqrt{5}}\left(1+\frac{1}{\phi^{2}}\right)
$$

and (5.10) follows easily.

Now apply (5.8) with $r=2 m-2$ and use the values from (5.9) to get that

$$
Q_{q m+m-2}= \begin{cases}\frac{1}{\sqrt{5}}\left(\phi^{q}-\bar{\phi}^{q}\right), & m \equiv 1,-1(\bmod 5), \\ \frac{1}{\sqrt{5}}\left(\phi^{q+2}-\bar{\phi}^{q+2}\right), & m \equiv 2,-2(\bmod 5) .\end{cases}
$$

If $m \equiv 1,-1(\bmod 5)$, then

$$
\frac{\phi^{q}}{\sqrt{5}}\left(1-\frac{1}{\phi^{4}}\right) \leq\left|Q_{q m+m-2}\right| \leq \frac{\phi^{q}}{\sqrt{5}}\left(1+\frac{1}{\phi^{4}}\right),
$$

and (5.11) follows. If $m \equiv 2,-2(\bmod 5)$, then

$$
\frac{\phi^{q+2}}{\sqrt{5}}\left(1-\frac{1}{\phi^{8}}\right) \leq\left|Q_{q m+m-2}\right| \leq \frac{\phi^{q+2}}{\sqrt{5}}\left(1+\frac{1}{\phi^{8}}\right)
$$

and (5.12) follows. Inequality (5.13) is an immediate consequence of the preceding inequalities.

Lemma 5. For $q \geq 2$,

$$
\frac{1}{\phi^{2 q+1}} \leq\left|K_{q m+m-1}(x)-K(x)\right| \leq \frac{1}{\phi^{2 q}}
$$




$$
\begin{gathered}
\frac{1}{\phi^{2 q-1}} \leq\left|K_{q m+m-2}(x)-K(x)\right| \leq \frac{1}{\phi^{2 q-2}}, \\
\max \left\{\left|R_{q m+m-1}(x)-R(x)\right|, \quad\left|R_{q m+m-2}(x)-R(x)\right|\right\} \leq \frac{1}{\phi^{2 q-6}} .
\end{gathered}
$$

Proof. Equation (5.7) implies that

$$
P_{q m+r}=F_{q} P_{m+r}+F_{q-1} P_{r}
$$

and

$$
Q_{q m+r}=F_{q} Q_{m+r}+F_{q-1} Q_{r} .
$$

From (․5.9) it follows that

$$
K_{q m+m-1}=\frac{P_{q m+m-1}}{Q_{q m+m-1}}=\frac{F_{q+1} P_{m-1}+F_{q}}{F_{q+1} Q_{m-1}} .
$$

Let $q \rightarrow \infty$ to deduce

$$
K(x)=\frac{P_{m-1} \phi+1}{Q_{m-1} \phi} .
$$

Since $\left|Q_{m-1}\right|=1$,

$$
\left|K_{q m+m-1}-K(x)\right|=\left|\frac{F_{q}}{F_{q+1}}-\frac{1}{\phi}\right|=\frac{\sqrt{5}}{\phi^{2 q+2}\left(1-\frac{(-1)^{q+1}}{\phi^{2 q+2}}\right)} .
$$

The last equality follows from Binet's formula (5.5). Thus for $q \geq 2$,

$$
\frac{\sqrt{5}}{\phi^{2 q+2}\left(1+\frac{1}{\phi^{6}}\right)} \leq\left|K_{q m+m-1}-K(x)\right| \leq \frac{\sqrt{5}}{\phi^{2 q+2}\left(1-\frac{1}{\phi^{6}}\right)} .
$$

Inequality (5.14) now follows.

Similarly,

$$
\frac{P_{q m+m-2}}{Q_{q m+m-2}}=\frac{P_{m-2} F_{q+1}}{Q_{m-2} F_{q+1}+F_{q}},
$$

and it follows that

$$
K(x)=\frac{P_{m-2} \phi}{Q_{m-2} \phi+1} .
$$

We consider the cases $m \equiv 1,-1(\bmod 5)$ and $m \equiv 2,-2(\bmod 5)$ separately. In the first case it can be seen from Table 1 that $Q_{m-2}=0$ and $\left|P_{m-2}\right|=1$. In this case,

$$
\left|K_{q m+m-2}-K(x)\right|=\left|\frac{F_{q+1}}{F_{q}}-\phi\right|=\frac{\sqrt{5}}{\phi^{2 q}\left(1-\frac{(-1)^{q}}{\phi^{q}}\right)} .
$$

Inequality (5.15) follows. For the second case, it can be seen from Table 1 that $Q_{m-2}=1$ and again $\left|P_{m-2}\right|=1$. In this case

$$
\left|K_{q m+m-2}-K(x)\right|=\left|\frac{F_{q+1}}{F_{q+2}}-\frac{1}{\phi}\right|=\frac{\sqrt{5}}{\phi^{2 q+4}\left(1-\frac{(-1)^{q+2}}{\phi^{2 q+4}}\right)},
$$

and (5.15) again follows. Inequality (5.16) follows from (5.14) and (5.15). 
Lemma 6. Let $q \geq 2$ and let $n=q m+m-1$ or $q m+m-2$. Let $y$ be another point on the unit circle. Suppose $P_{n}(y)=P_{n}(x)+\epsilon_{1}, Q_{n}(y)=Q_{n}(x)+\epsilon_{2}$, with $\epsilon=\max \left\{\left|\epsilon_{1}\right|,\left|\epsilon_{2}\right|\right\}<1 / 2$. Then

$$
\left|K_{n}(y)-K_{n}(x)\right| \leq \frac{10 \epsilon}{\phi^{q-2}} .
$$

If $q \geq 3$ and the angle between $x$ and $y$ (measured from the origin) is less than $5 \pi / 3$ and $\epsilon \leq 1 /\left(20 \phi^{2}\right)$, then

$$
\left|R_{n}(y)-R_{n}(x)\right|<3 \phi|x-y|+\frac{60 \epsilon}{\phi^{q-4}}
$$

and

$$
\left|R_{n}(y)-R(x)\right| \leq 3 \phi|x-y|+\frac{60 \epsilon}{\phi^{q-4}}+\frac{1}{\phi^{2 q-6}} .
$$

Proof. From the statement of the conditions in the lemma, we have that

$$
\begin{aligned}
\left|K_{n}(y)-K_{n}(x)\right| & =\left|\frac{P_{n}(y)}{Q_{n}(y)}-\frac{P_{n}(x)}{Q_{n}(x)}\right|=\left|\frac{\epsilon_{1} Q_{n}(x)-\epsilon_{2} P_{n}(x)}{Q_{n}(x)\left(Q_{n}(x)+\epsilon_{2}\right)}\right| \\
& \leq \frac{\left|\epsilon_{1}-\epsilon_{2}\right|}{\left|Q_{n}(x)+\epsilon_{2}\right|}+\frac{\left|\epsilon_{2}\right|\left|P_{n}(x)-Q_{n}(x)\right|}{\left|Q_{n}(x)\right|\left|Q_{n}(x)+\epsilon_{2}\right|} \\
& =\frac{\left|\epsilon_{1}-\epsilon_{2}\right|}{\left|Q_{n}(x)+\epsilon_{2}\right|}+\frac{\left|\epsilon_{2}\right|\left|K_{n}(x)-1\right|}{\left|Q_{n}(x)+\epsilon_{2}\right|} \\
& \leq \frac{2 \epsilon}{|| Q_{n}(x)|-\epsilon|}+\frac{\epsilon|| K(x)\left|+1 / \phi^{2 q-2}+1\right|}{|| Q_{n}(x)|-\epsilon|} .
\end{aligned}
$$

Here we have used (5.14), (5.15) and the bounds on $\epsilon_{1}$ and $\epsilon_{2}$ given in the statement of the lemma. Since $|K(x)| \leq \phi$ and $\epsilon<1 / 2$, it follows that

$$
\begin{aligned}
\left|K_{n}(y)-K_{n}(x)\right| & \leq \frac{2 \epsilon}{|| Q_{n}(x)|-1 / 2|}+\frac{3 \epsilon}{|| Q_{n}(x)|-1 / 2|} \\
& =\frac{5 \epsilon}{|| Q_{n}(x)|-1 / 2|} \\
& \leq \frac{10 \epsilon}{\phi^{q-2}} .
\end{aligned}
$$

The last inequality follows from (5.10), (5.11), 5.12). Similarly,

$$
\begin{aligned}
\left|R_{n}(y)-R_{n}(x)\right| & =\left|\frac{y^{1 / 5}}{K_{n}(y)}-\frac{x^{1 / 5}}{K_{n}(x)}\right| \\
& =\left|\frac{K_{n}(x)\left(y^{1 / 5}-x^{1 / 5}\right)+x^{1 / 5}\left(K_{n}(x)-K_{n}(y)\right)}{K_{n}(x) K_{n}(y)}\right|
\end{aligned}
$$




$$
\begin{aligned}
& \leq \frac{|x-y|}{\left|K_{n}(y)\right|}+\frac{\left|K_{n}(x)-K_{n}(y)\right|}{\left|K_{n}(x)\right|\left|K_{n}(y)\right|} \\
& \leq \frac{|x-y|}{|| K_{n}(x)\left|-10 \epsilon / \phi^{q-2}\right|}+\frac{10 \epsilon / \phi^{q-2}}{\left|K_{n}(x)\right||| K_{n}(x)\left|-10 \epsilon / \phi^{q-2}\right|} .
\end{aligned}
$$

Here we have used (5.17) and the fact that the bound on the angle between $x$ and $y$ implies that $\left|y^{1 / 5}-x^{1 / 5}\right| \leq|x-y|$ (since this bound implies $\left|1-(y / x)^{1 / 5}\right| \leq|1-y / x|$ ). From (5.14), (5.15) and the bound on $\epsilon$, it follows that

$$
\begin{aligned}
\left|R_{n}(y)-R_{n}(x)\right| \leq & \frac{|x-y|}{|| K(x)\left|-1 / \phi^{2 q-2}-1 /\left(2 \phi^{q}\right)\right|} \\
& +\frac{10 \epsilon}{\phi^{q-2}|| K(x)\left|-1 / \phi^{2 q-2}\right||| K(x)\left|-1 / \phi^{2 q}-1 / 2 \phi^{q}\right|} .
\end{aligned}
$$

Since $|K(x)|=\phi$ or $1 / \phi$, it follows that

$$
\begin{aligned}
&\left|R_{n}(y)-R_{n}(x)\right| \leq \frac{|x-y| \phi}{1-1 / \phi^{2 q-3}-1 /\left(2 \phi^{q-1}\right)} \\
&+\frac{10 \epsilon}{\phi^{q-4}\left(1-1 / \phi^{2 q-3}\right)\left(1-1 / \phi^{2 q-1}-1 /\left(2 \phi^{q-1}\right)\right)} \\
& \leq \frac{|x-y| \phi}{1-1 / \phi^{3}-1 /\left(2 \phi^{2}\right)} \\
& \quad+\frac{10 \epsilon}{\phi^{q-4}\left(1-1 / \phi^{3}\right)\left(1-1 / \phi^{3}-1 /\left(2 \phi^{2}\right)\right)} \\
& \leq 3 \phi|x-y|+\frac{60 \epsilon}{\phi^{q-4}} .
\end{aligned}
$$

Finally, (5.19) follows from (5.16) and (5.18).

Lemma 7. There exists an uncountable set of points on the unit circle such that if $y$ is one of these points, then there exist two increasing sequences of integers, $\left\{n_{i}\right\}_{i=1}^{\infty}$ and $\left\{m_{i}\right\}_{i=1}^{\infty}$ say, such that

$$
\begin{aligned}
& \lim _{i \rightarrow \infty} R_{n_{i}}(y)=\lim _{i \rightarrow \infty} R_{n_{i}-1}(y)=R_{a}, \\
& \lim _{i \rightarrow \infty} R_{m_{i}}(y)=\lim _{i \rightarrow \infty} R_{m_{i}-1}(y)=R_{b},
\end{aligned}
$$

for some $a, b \in\{1,2, \ldots, 10\}$, where $a \neq b$.

Proof. With the notation of Theorem 4, let $t \in S^{\diamond}$ and set $y=\exp (2 \pi i t)$. Let $c_{f_{n}} / d_{f_{n}}$ be one of the infinitely many approximants satisfying (5.1) and (5.2), and set $x_{n}=\exp \left(2 \pi i c_{f_{n}} / d_{f_{n}}\right)$. Then $R\left(x_{n}\right)=R_{a}$ and

$$
\left|x_{n}-y\right|<\frac{1}{d_{f_{n}}^{2}\left(d_{f_{n}}+1\right)^{2} \phi^{d_{f_{n}}^{2}+2 d_{f_{n}}}} .
$$

For the last inequality we have used the condition on the $e_{h_{n}+1}$ 's in (5.2) in the same way that the condition on the $e_{i+1}(t)$ 's in (2.9) was used in Lemma 1, and 
the fact that chord length is shorter than arc length. Let $k=d_{f_{n}}^{2}+d_{f_{n}}-1$ or $d_{f_{n}}^{2}+d_{f_{n}}-2$. By (2.5), (2.6) and (5.20) it follows that

$$
\left|P_{k}\left(x_{n}\right)-P_{k}(y)\right| \leq \frac{1}{\phi^{d_{f_{n}}}}
$$

and

$$
\left|Q_{k}\left(x_{n}\right)-Q_{k}(y)\right| \leq \frac{1}{\phi^{d_{f_{n}}}} .
$$

By (5.19), with $k$ as above, $q=m=d_{f_{n}}$ and $\epsilon=1 / \phi^{d_{f_{n}}}$, it follows that

$$
\begin{aligned}
& \left|R_{k}(y)-R_{a}\right|=\left|R_{k}(y)-R\left(x_{n}\right)\right| \\
& \leq \frac{3 \phi}{d_{f_{n}}^{2}\left(d_{f_{n}}+1\right)^{2} \phi^{d_{f_{n}}^{2}+2 d_{f_{n}}}}+\frac{60}{\phi^{2 d_{f_{n}}-4}}+\frac{1}{\phi^{2 d_{f_{n}}-3}} \leq \frac{500}{\phi^{2 d_{f_{n}}}} .
\end{aligned}
$$

Thus

$$
\lim _{n \rightarrow \infty} R_{d_{f_{n}}^{2}+d_{f_{n}}-1}(y)=\lim _{n \rightarrow \infty} R_{d_{f_{n}}^{2}+d_{f_{n}}-2}(y)=R_{a}
$$

Similarly,

$$
\lim _{n \rightarrow \infty} R_{d_{g_{n}}^{2}+d_{g_{n}}-1}(y)=\lim _{n \rightarrow \infty} R_{d_{g_{n}}^{2}+d_{g_{n}}-2}(y)=R_{b}
$$

It is clear that $S^{\diamond}$ is an uncountable set, and thus $G=\left\{\exp (2 \pi i t): t \in S^{\diamond}\right\}$ is an uncountable set.

Proof of Theorem 母 Let $y$ be any point in $G$, where $G$ is defined in the proof of Lemma 7, and let $t$ be the irrational in $(0,1)$ for which $y=\exp (2 \pi i t)$.

Suppose $R(y)$ converges generally to $f \in \hat{\mathbb{C}}$ and that $\left\{v_{n}\right\},\left\{w_{n}\right\}$ are two sequences such that

$$
\lim _{n \rightarrow \infty} \frac{P_{n}+v_{n} P_{n-1}}{Q_{n}+v_{n} Q_{n-1}}=\lim _{n \rightarrow \infty} \frac{P_{n}+w_{n} P_{n-1}}{Q_{n}+w_{n} Q_{n-1}}=\frac{y^{\frac{1}{5}}}{f}:=g .
$$

Suppose first that $|g|<\infty$. By construction there exist two infinite strictly increasing sequences of positive integers $\left\{n_{i}\right\}_{i=1}^{\infty},\left\{m_{i}\right\}_{i=1}^{\infty} \subset \mathbb{N}$ such that

$$
L_{a}:=\frac{y^{\frac{1}{5}}}{R_{a}}=\lim _{i \rightarrow \infty} \frac{P_{n_{i}}(y)}{Q_{n_{i}}(y)}=\lim _{i \rightarrow \infty} \frac{P_{n_{i}-1}(y)}{Q_{n_{i}-1}(y)}
$$

and

$$
L_{b}:=\frac{y^{\frac{1}{5}}}{R_{b}}=\lim _{i \rightarrow \infty} \frac{P_{m_{i}}(y)}{Q_{m_{i}}(y)}=\lim _{i \rightarrow \infty} \frac{P_{m_{i}-1}(y)}{Q_{m_{i}-1}(y)}
$$

for some $a \neq b, a, b \in\{1,2, \ldots, 10\}$. Also by construction each $n_{i}$ has the form $d_{k_{i}}^{2}+d_{k_{i}}-1$, where $d_{k_{i}}$ is some denominator convergent in the continued fraction expansion of $t$. Each $m_{i}$ has a similar form. It can be further assumed that $L_{a} \neq g$, since $L_{a} \neq L_{b}$. For ease of notation, write

$$
\begin{array}{ll}
P_{n_{i}}(y)=P_{n_{i}}, & Q_{n_{i}}(y)=Q_{n_{i}}, \\
P_{n_{i}-1}(y)=P_{n_{i}-1}, & Q_{n_{i}-1}(y)=Q_{n_{i}-1} .
\end{array}
$$


Write $P_{n_{i}}=Q_{n_{i}}\left(L_{a}+\epsilon_{n_{i}}\right)$ and $P_{n_{i}-1}=Q_{n_{i}-1}\left(L_{a}+\delta_{n_{i}}\right)$, where $\epsilon_{n_{i}} \rightarrow 0$ and $\delta_{n_{i}} \rightarrow 0$ as $i \rightarrow \infty$. Thus

$$
\frac{Q_{n_{i}}\left(L_{a}+\epsilon_{n_{i}}\right)+w_{n_{i}} Q_{n_{i}-1}\left(L_{a}+\delta_{n_{i}}\right)}{Q_{n_{i}}+w_{n_{i}} Q_{n_{i}-1}}=g+\gamma_{n_{i}},
$$

where $\gamma_{n_{i}} \rightarrow 0$ as $i \rightarrow \infty$. This last equation implies that

$$
w_{n_{i}}+\frac{Q_{n_{i}}}{Q_{n_{i}-1}}=\frac{Q_{n_{i}}}{Q_{n_{i}-1}} \times \frac{\epsilon_{n_{i}}-\delta_{n_{i}}}{g-L_{a}+\gamma_{n_{i}}-\delta_{n_{i}}} .
$$

Because of (5.13), the fact that each $n_{i}$ has the form $d_{k_{i}}^{2}+d_{k_{i}}-1$, where $d_{k_{i}}$ is some denominator convergent in the continued fraction expansion of $t$, and (5.22), it follows that $Q_{n_{i}} / Q_{n_{i}-1}$ is absolutely bounded. Therefore the right hand side of the last equality tends to 0 as $i \rightarrow \infty$, and thus, as $n_{i} \rightarrow \infty$,

$$
w_{n_{i}}+Q_{n_{i}} / Q_{n_{i}-1} \rightarrow 0 .
$$

Note that $\left|w_{n_{i}}\right|<\infty$ for all $i$ sufficiently large, since $\left|Q_{n_{i}} / Q_{n_{i}-1}\right|<\infty$. Similarly,

$$
\frac{Q_{n_{i}}\left(L_{a}+\epsilon_{n_{i}}\right)+v_{n_{i}} Q_{n_{i}-1}\left(L_{a}+\delta_{n_{i}}\right)}{Q_{n_{i}}+v_{n_{i}} Q_{n_{i}-1}}=g+\mu_{n_{i}}
$$

where $\mu_{n_{i}} \rightarrow 0$ as $i \rightarrow \infty$. This in turn implies that

$$
v_{n_{i}}+Q_{n_{i}} / Q_{n_{i}-1} \rightarrow 0
$$

as $n_{i} \rightarrow \infty$. By (5.28), (5.30) and the triangle inequality, $\lim _{i \rightarrow \infty}\left|v_{n_{i}}-w_{n_{i}}\right|=0$. Thus $\liminf d\left(v_{n}, w_{n}\right)=0$.

Therefore $R(y)$ does not converge generally. If $g=\infty$, then replace $g+\gamma_{n_{i}}$ on the right side of (5.26) by $1 / \gamma_{n_{i}}$, and $g-L_{a}+\gamma_{n_{i}}$ in the denominator of the right side of (5.27) by $-L_{a}+1 / \gamma_{n_{i}}$, where $\gamma_{n_{i}} \rightarrow 0$ as $i \rightarrow \infty$, and the remainder of the argument is the same.

Since $G$ is uncountable, this proves the theorem.

Remark. $G$ is clearly of measure 0 , as it is a subset of the set $\{y: y=\exp (2 \pi i t), t \in$ $S$ \}, where $S$ is the set from Theorem [2]

Corollary 3, Let $y$ be as in Corollary 1, Then $K(y)$ does not converge generally.

Proof. Let $t \in(0,1)$ be such that $y=\exp (2 \pi i t)$. Recall that $t=\left[0, a_{1}, a_{2}, \ldots\right]$, where $a_{i}$ is the integer consisting of a tower of $i$ twos with an $i$ an top. Modulo 5, the approximants in the continued fraction expansion of $t$ are

$$
\left\{\frac{0}{1}, \frac{1}{2}, \frac{1}{3}, \frac{2}{0}, \frac{3}{3}, \frac{0}{3}, \frac{3}{1}, \frac{3}{4}, \frac{1}{0}, \frac{4}{4}, \frac{0}{4}, \frac{4}{3}, \frac{4}{2}, \frac{3}{0}, \frac{2}{2}, \frac{0}{2}, \frac{2}{4}, \frac{2}{1}, \frac{4}{0}, \frac{1}{1}\right\},
$$

where once again the bar indicates that the approximants repeat modulo 5 in this order. In particular, there are two fractions, $r / s$ and $u / v$, say, such that (5.1) and (5.3) hold. Thus it is sufficient to show that

$$
\left|t-\frac{c_{i}}{d_{i}}\right|<\frac{1}{2 \pi d_{i}^{2}\left(d_{i}+1\right)^{2} \phi^{d_{i}^{2}+2 d_{i}}},
$$

for all $i \geq 3$, where $c_{i} / d_{i}$ is the $i$-th approximant in the continued fraction expansion of $t$. In particular, (15.20) will hold, where $\left\{c_{f_{n}} / d_{f_{n}}\right\}$ is the sequence of approximants corresponding to $r / s$. A similar inequality holds for $\left\{c_{g_{n}} / d_{g_{n}}\right\}$, where $\left\{c_{g_{n}} / d_{g_{n}}\right\}$ is the sequence of approximants corresponding to $u / v$. This in turn will ensure that 
$y \in G$, so that $K(y)$ will not converge generally by Theorem 4 We will show that, for $i \geq 3$,

$$
e_{i+1}>16^{d^{2}}
$$

This will be sufficient to prove the result. Indeed, let $t_{i+1}=\left[e_{i+1}, e_{i+2}, \ldots\right]$ denote the $i$-th tail of the continued fraction expansion for $t$. Then

$$
\begin{aligned}
e_{i+1} & \geq 16^{d_{i}^{2}}=4^{2 \cdot d_{i}^{2}}>4^{\left(d_{i}+1\right)^{2}} \\
& =2^{\left(d_{i}+1\right)^{2}}{ }_{2}\left(d_{i}+1\right)^{2}>2 \pi\left(d_{i}+1\right)^{2} \phi^{d_{i}^{2}+2 d_{i}}
\end{aligned}
$$

This implies that

$$
\begin{aligned}
\left|t-\frac{c_{i}}{d_{i}}\right| & =\left|\frac{t_{i+1} c_{i}+c_{i-1}}{t_{i+1} d_{i}+d_{i-1}}-\frac{c_{i}}{d_{i}}\right|=\frac{1}{d_{i}\left(t_{i+1} d_{i}+d_{i-1}\right)} \\
& <\frac{1}{d_{i}\left(e_{i+1} d_{i}+d_{i-1}\right)}<\frac{1}{d_{i}^{2} e_{i+1}}<\frac{1}{2 \pi d_{i}^{2}\left(d_{i}+1\right)^{2} \phi^{d_{i}^{2}+2 d_{i}}} .
\end{aligned}
$$

Thus all that remains is to prove (5.33). The proof of this inequality is similar to that of (2.13). To that end,

$$
16^{d_{i}^{2}} \leq e_{i+1} \text { if and only if } 4 d_{i}^{2} \leq \underbrace{2^{2^{i+1}}}_{i \text { twos }},
$$

where the notation indicates that the last integer consists of a tower of $i$ twos with an $i+1$ on top. It can be easily checked that the second inequality holds for $i=3,4$. Suppose it holds for $i=3,4, \ldots, r-1$. Then

$$
\begin{aligned}
4 d_{r}^{2} & =4\left(e_{r} d_{r-1}+d_{r-2}\right)^{2} \leq 4\left(4 e_{r} d_{r-1}^{2}+4 d_{r-2}^{2}\right)^{2} \\
& \left.\leq 4(4 \times \underbrace{2^{2^{r}}}_{r \text { twos }} \times \underbrace{2^{2}}_{(r-1) \text { twos }}+4 \times \underbrace{2^{r}}_{(r-2) \text { twos }})^{2^{r-1}}\right)^{2} \\
& \leq \underbrace{2^{2^{r+1}}}_{r \text { twos }} .
\end{aligned}
$$

Thus the first inequality in (5.34) holds for all positive integers $i \geq 3$, and the result follows.

\section{The NATURE OF THE NON-CONVERGENCE ON THE UNIT CIRCLE}

Proposition 1. There exists an uncountable set $G^{*}$ of points on the unit circle such that if $y \in G^{*}$, then there exist ten sequences of positive integers $\left\{n_{i, j}\right\}_{i=1}^{\infty}$, $1 \leq j \leq 10$, such that $\lim _{i \rightarrow \infty} R_{n_{i, j}}(y)=R_{j}$.

Proof. The proof is similar to that of Lemma 7 . Let

$$
W=\left\{W_{i}\right\}_{i=1}^{12}=\left\{R_{6}, R_{7}, R_{8}, R_{9}, R_{10}, R_{2}, R_{3}, R_{4}, R_{5}, R_{1}, R_{8}, R_{7}\right\} .
$$


Note that $W$ contains all ten of the values taken by the Rogers-Ramanujan continued fraction at roots of unity. Consider the continued fraction

$$
\alpha=\left[0,1,3, \overline{2,3,2,1,1,2,3,2,1,3,3,5]}:=\left[0, a_{1}, a_{2}, \ldots\right] .\right.
$$

Modulo 5, the approximants are

$$
\left\{\frac{0}{1}, \overline{\frac{1}{1}}, \frac{3}{4}, \frac{2}{4}, \frac{4}{1}, \frac{0}{1}, \frac{4}{2}, \frac{4}{3}, \frac{2}{3}, \frac{0}{2}, \frac{2}{2}, \frac{2}{4}, \frac{3}{4}\right\}
$$

where the bar indicates that, modulo 5 , the approximants repeat in this order.

Let $t$ be any irrational in $(0,1)$ such that, for $i \geq 1$, the $i$-th partial quotient $b_{i}$ and the $i$-th approximant $c_{i} / d_{i}$ in its continued fraction expansion $\left[0, b_{1}, b_{2}, \ldots\right]$ satisfy the following conditions:

$$
\begin{aligned}
& \text { (i) } b_{i} \equiv a_{i}(\bmod 5), \\
& \text { (ii) }\left|t-\frac{c_{i}}{d_{i}}\right|<\frac{1}{2 \pi d_{i}^{2}\left(d_{i}+1\right)^{2} \phi^{d_{i}^{2}+2 d_{i}}},
\end{aligned}
$$

where the $a_{i}$ 's are as in (6.1). Set $y=\exp (2 \pi i t)$ and let $x_{n}=\exp \left(2 \pi i c_{n} / d_{n}\right)$, so that

$$
\left|x_{n}-y\right|<\frac{1}{d_{n}^{2}\left(d_{n}+1\right)^{2} \phi^{d_{n}^{2}+2 d_{n}}} .
$$

Here we once again have used the fact that chord length is less than arc length. Set $r=n(\bmod 12)$, for $n>0$. Then it can be easily checked, using (1.4) and (6.2), that

$$
R\left(x_{n}\right)= \begin{cases}W_{r}, & r \neq 0 \\ W_{12} & r=0 .\end{cases}
$$

Let $k=d_{n}^{2}+d_{n}-1$ or $d_{n}^{2}+d_{n}-2$. By (2.5), (2.6) and (6.4), it follows that

$$
\left|P_{k}\left(x_{n}\right)-P_{k}(y)\right| \leq \frac{1}{\phi^{d_{n}}}
$$

and

$$
\left|Q_{k}\left(x_{n}\right)-Q_{k}(y)\right| \leq \frac{1}{\phi^{d_{n}}} .
$$

By (5.19), with $k$ as above, $q=m=d_{n}$ and $\epsilon=1 / \phi^{d_{n}}$, it follows that

$$
\begin{aligned}
\left|R_{k}(y)-R\left(x_{n}\right)\right| & \leq \frac{3 \phi}{d_{n}^{2}\left(d_{n}+1\right)^{2} \phi^{d_{n}^{2}+2 d_{n}}}+\frac{60}{\phi^{2 d_{n}-4}}+\frac{1}{\phi^{2 d_{n}-3}} \\
& \leq \frac{500}{\phi^{2 d_{n}}} .
\end{aligned}
$$

Next, for each $j \in\{1,2, \ldots, 12\}$, define a sequence of integers $\left\{s_{i, j}\right\}_{i=1}^{\infty}$ by setting $s_{i, j}=d_{12(i-1)+j}^{2}+d_{12(i-1)+j}$. By (6.5), $R\left(x_{12(i-1)+j}\right)=W_{j}$, and so, from (6.7),

$$
\begin{gathered}
\left|R_{\left(s_{i, j}-1\right)}(y)-W_{j}\right| \leq \frac{500}{\phi^{2 d_{12(i-1)+j}}}, \\
\left|R_{\left(s_{i, j}-2\right)}(y)-W_{j}\right| \leq \frac{500}{\phi^{2 d_{12(i-1)+j}}} .
\end{gathered}
$$


It follows that

$$
\lim _{i \rightarrow \infty} R_{\left(s_{i, j}-1\right)}(y)=\lim _{i \rightarrow \infty} R_{\left(s_{i, j}-2\right)}(y)=W_{j} .
$$

Both results hold for $1 \leq j \leq 12$. Since the set $W$ contains all ten of the $R_{j}$ 's, the result is proved for this particular $t$.

Let $S^{\prime}$ denote the set of all such $t \in(0,1)$ and set $G^{*}=\left\{\exp (2 \pi i t): t \in S^{\prime}\right\}$. Clearly $G^{*} \subset Y_{S}$ and is also uncountable.

Corollary 2. Let the sequence of integers $\left\{a_{i}\right\}_{i=1}^{\infty}$ be defined by

$$
\left\{a_{1}, a_{2}, \cdots\right\}=\{0,2, \overline{1,2,1,0,0,1,2,1,0,2,2,4}\},
$$

where the bar indicates that the sequence under it repeats infinitely often. Let $t$ be the number with regular continued fraction expansion given by

$$
t=\left[0, g_{1}+a_{1}, g_{2}+a_{2}, g_{3}+a_{3}, \cdots\right]
$$

where $g_{i}$ is the integer consisting of a tower of $i$ sixteens with an $i$ an top and the $a_{i}$ 's are as above, i.e.,

$$
t=\left[0,16,16^{16^{2}}+2,16^{16^{16^{3}}}+1, \cdots\right] .
$$

If $y=\exp (2 \pi i t)$, then $R(y)$ has subsequences of approximants tending to all ten values taken by the Rogers-Ramanujan continued fraction at roots of unity.

Proof. Showing that the $i$-th partial quotient $b_{i}$ and the $i$-th approximant $c_{i} / d_{i}$ of the continued fraction expansion of $t$ satisfy the conditions in (6.3), for $i=1,2, \cdots$, will ensure that $y \in G^{*}$, where $G^{*}$ is defined in Proposition 1 .

The $b_{i}$ 's satisfy the first of these conditions by construction, and so it remains to prove the second. By the same reasoning as used in the proof of Corollary 3 it is sufficient to show that

$$
g_{i+1} \geq 16^{d_{i}^{2}}
$$

since $b_{i+1} \geq g_{i+1}$. The details are omitted since the proof is almost identical, the only real difference being that

$$
16^{d_{i}^{2}} \leq g_{i+1} \text { if and only if } d_{i}^{2} \leq \underbrace{10^{16^{i+1}}}_{i \times 16^{\prime} \mathrm{s}} .
$$

\section{Concluding Remarks}

The set of points on the unit circle for which we have shown that the RogersRamanujan continued fraction diverges has measure zero. This still leaves open the question of convergence for the remaining points. We conjecture that the RogersRamanujan continued fraction diverges, in the classical sense and the general sense, almost everywhere on the unit circle.

In later papers we will examine the question of convergence, on and off the unit circle, of other $q$-continued fractions such as the Göllnitz-Gordon continued fraction and certain q-continued fractions studied by Ramanujan and Selberg. 


\section{REFERENCES}

[1] Andrews, G. E.; Berndt, Bruce C.; Jacobsen, Lisa; Lamphere, Robert L. The continued fractions found in the unorganized portions of Ramanujan's notebooks. Mem. Amer. Math. Soc. 99 (1992), no. 477, vi+71pp MR 93f:11008

[2] Berndt, Bruce C.; Chan, Heng Huat; Zhang, Liang-Cheng Explicit evaluations of the RogersRamanujan continued fraction. J. Reine Angew. Math. 480 (1996), 141-159. MR 98c:11007

[3] Berndt, Bruce C.; Chan, Heng Huat Some values for the Rogers-Ramanujan continued fraction. Canad. J. Math. 47 (1995), no. 5, 897-914. MR 97a:33043

[4] Borwein, Peter; Erdélyi, Tamás, Polynomials and polynomial inequalities, Springer, New York, 1995. x+48 0 pp. MR 97e:41001

[5] Hardy, G. H. (Godfrey Harold). Lectures by Godfrey H. Hardy on the mathematical work of Ramanujan; fall term 1936 / Notes by Marshall Hall. The Institute for Advanced Study. Ann Arbor, Mich., Edwards Bros., Inc., 1937.

[6] Huang, Sen-Shan. Ramanujan's evaluations of Rogers-Ramanujan type continued fractions at primitive roots of unity. Acta Arith. 80 (1997), no. 1, 49-60. MR 98h:11012

[7] Jacobsen, Lisa General convergence of continued fractions. Trans. Amer. Math. Soc. 294 (1986), no. 2, 477-485. MR 87j:40004

[8] Lorentzen, Lisa; Waadeland, Haakon Continued fractions with applications. Studies in Computational Mathematics, 3. North-Holland Publishing Co., Amsterdam, 1992, 35-36, 67-68. MR 93g:30007

[9] Milovanović, G. V; Mitrinović, D. S; Rassias, Th. M; Topics in polynomials: extremal problems, inequalities, zeros, World Sci. Publishing, River Edge, NJ, 1994. xiv+821 pp. MR 95m:30009

[10] Ramanujan, S. Notebooks (2 volumes), Tata Institute of Fundamental Research, Bombay, 1957. MR 20:6340

[11] Ramanujan, S. Collected Papers, Chelsea, New York, 1962.

[12] Ramanathan, K. G. On Ramanujan's continued fraction. Acta Arith. 43 (1984), no. 3, 209226 MR 85d:11012

[13] Rockett, Andrew M.; Szüsz, Peter, Continued fractions. World Scientific Publishing Co., Inc., River Edge, NJ, 1992, pp 140-141. MR 93m:11060

[14] L.J.Rogers, Second memoir on the expansion of certain infinite products, Proc. London Math. Soc. 25 (1894), 318-343.

[15] Schur, Issai, Ein Beitrag zur additiven Zahlentheorie und zur Theorie der Kettenbrüchen, in Gesammelte Abhandlungen. Band II, Springer-Verlag, Berlin-New York, 1973, 117-136. (Originally in Sitzungsberichte der Preussischen Akadamie der Wissenschaften, 1917, Physikalisch-Mathematische Klasse, 302-321) MR 57:2858b

[16] Yi,J. Evaluation of the Rogers-Ramanujan continued fraction $R(q)$ by modular equations, Acta Arith. 98 (2001), no. 3, 103-127. MR 2002i:11021

Department of Mathematical Sciences, Northern Illinois University, DeKalb, IlliNOIS 60115

E-mail address: bowman@math.niu.edu

Department of Mathematics, Trinity College, 300 Summit Street, Hartford, ConNECTICUT 06106-3100

E-mail address: james.mclaughlin@trincoll.edu 